\title{
Renormalized time scale for anticipating and lagging synchronization
}

Article

Accepted Version

Hayashi, Y., Nasuto, S. J. and Eberle, H. (2016) Renormalized time scale for anticipating and lagging synchronization. Physical Review E, 93 (5). 052229. ISSN 2470-0053 doi: https://doi.org/10.1103/PhysRevE.93.052229 Available at https://centaur.reading.ac.uk/65650/

It is advisable to refer to the publisher's version if you intend to cite from the work. See Guidance on citing.

To link to this article DOI: http://dx.doi.org/10.1103/PhysRevE.93.052229

Publisher: American Physical Society

All outputs in CentAUR are protected by Intellectual Property Rights law, including copyright law. Copyright and IPR is retained by the creators or other copyright holders. Terms and conditions for use of this material are defined in the End User Agreement.

\section{www.reading.ac.uk/centaur}

\section{CentAUR}

Central Archive at the University of Reading

Reading's research outputs online 


\title{
Renormalized time scale for anticipating and lagging synchronization
}

\author{
Yoshikatsu Hayashi*, Slawomir J. Nasuto, Henry Eberle \\ Brain Embodiment Lab, School of Systems Engineering/Biological Sciences, \\ University of Reading, Reading RG6 6AY, United Kingdom*
}

(Dated: May 17, 2016)

\begin{abstract}
Anticipating synchronization has been recently proposed as a mechanism of interaction in dynamical systems which are able to bring about predictions of future states of a driver system. We suggest that an interesting insight into the anticipating synchronization can be obtained by the renormalization of the time scale in the driven system. Our approach directly links the feedback delay of the driven system with the renormalized time scale of the driven system, identifying the main component in the anticipating synchronization paradigm and suggesting an alternative method to generate the anticipating and the lagging synchronization.
\end{abstract}

PACS numbers: 05.45.Xt, 05.45.Pq

\section{INTRODUCTION}

Synchronization of oscillations is abundant in nature from physical, chemical to biological systems $[1,2]$. The synchronization of chaotic systems is a surprising phenomenon considering the exponential divergence of the trajectories, and chaotic synchronization as a new subfield of nonlinear systems has been increasingly investigated in the past two decades [3].

Anticipating synchronization has been recently proposed as a mechanism of interaction in dynamical systems able to bring about predictions of future states of a driver system by a driven system. A general framework was identified for anticipation by the driven system with delay [4]:

$$
\begin{aligned}
& \dot{x}(t)=f[x(t)] \\
& \dot{y}(t)=f[y(t)]+K[x(t)-y(t-\tau)],
\end{aligned}
$$

where $f(\cdot)$ describes the internal dynamics of the state variables of both the driver system, $x$, and the driven system, $y$. Note here that the state variables can be extended to the multidimensional state variables. The second term in the driven system is a coupling term which drives it to be synchronized with the driver system. The term $y(t-\tau)$ takes into account the feedback delay in the driven system referring to its own state.

The anticipating synchronization is simply identified by the condition that manifold, $y(t)=x(t+\tau)$ be stable, hereafter, it will be called anticipating manifold. This implies that the driven system is anticipating the future state of the driver system.

This paradigm of anticipating synchronization in a unidirectional coupling configuration has been investigated in a variety of systems, chaotic lasers [5], autonomous chaotic systems [6], and excitable systems in the noisy environment $[7,8]$ numerically and experimentally. The

\footnotetext{
*Electronic address: y.hayashi@reading.ac.uk; URL: http:// bel.reading.ac.uk/
}

paradigm was also extended to excitable systems in order to develop a control method to predict the driver's behavior, and to prevent the driver from firing [9]. Interestingly, it is the presence of a delay in the coupling that is a necessary condition to achieve the driven system's anticipation of the driver's dynamics. Pointing out the mechanism of the anticipating synchronization, the lowering of the excitability threshold of the slave in a delayed coupling scheme was shown to be a general mechanism for anticipating synchronization in excitable systems [8], and the linear stability analysis of the simple linear systems has been performed [10], and the effectiveness of the first order expansion was explored as a proof of concept [11].

In this paper, the paradigm of the anticipating synchronization is explored, leading to essential understanding of how the time delay gives rise to anticipating synchronization of the driven system, and we propose an alternative method of renormalized time step to generate the anticipating synchronization. The positive status of the delayed feedback is shown in a straightforward manner, i.e., the first order approximation of the coupling term of the driven system leads to the renormalization of time, amounting to speeding up the evolution of the driven system. On the other hand, the renormalization of time can also lead to the lagging synchronization by slowing down the evolution of the driven system. The chaotic system and the excitable systems were used to numerically validate the anticipating and lagging synchronization based on the renormalization of time.

\section{ANALYTICAL SOLUTIONS FOR ONE DIMENSIONAL LINEAR CASE}

\section{A. First order expansion of the time delay term}

Consider a linear stability analysis for the anticipating manifold, taking a linear system, $f(x)=\alpha x$. First, we shift the time of the driver system by $\tau$ in Eq. (1). 
Subtracting Eq. (1) from Eq. (2), we have

$$
\dot{z}(t)=\alpha z(t)-K z(t-\tau),
$$

defining the transversal system, $z(t)=x(t+\tau)-y(t)$. As a next step, we expand the delay term, $z(t-\tau)$ in $\tau$ to the first order. After rearranging of the terms, the equation (3) becomes

$$
\dot{z}(t)=-\frac{K-\alpha}{1-K \tau} z(t) .
$$

Equation (4) specifies two conditions for the presence of anticipating manifold as follows;

$$
\begin{aligned}
K & >\alpha, \\
K \tau & <1 .
\end{aligned}
$$

These two conditions also indicate that the anticipatory horizon, the maximum $\tau$ value in the $\tau-K$ plane should be given by the intersection of $K \tau=1$ and $K=\alpha$.

On the other hand, when $\tau<0$, Equation (4) specifies one condition for the presence of anticipating manifold as follows;

$$
K>\alpha
$$

\section{B. Solution for the delay differential equation}

The stability conditions for the linear systems with the delay term can be obtained by calculating complex Lyapunov exponent. Extending the state variable to the complex plane, the stability conditions for the anticipating manifold can be calculated for the explicit time delay. In contrast to the section II A. where the solution for the driver dynamics is given by $x=A e^{\alpha t}$, the solution should be given by $x=A e^{(\alpha+i \omega) t}$. Thus, the driver dynamics follows $\dot{x}(t)=(\alpha+i \omega) x(t)$. The corresponding driven dynamics is given by $\dot{y}(t)=(\alpha+i w) y(t)+$ $K e^{i \beta}[x(t)-y(t-\tau)]$ where the coupling constant is also given by the complex number, $K e^{i \beta}$. The transversal system, $z(t)=x(t+\tau)-y(t)$ evolves as

$$
\dot{z}(t)=(\alpha+i w) z(t)-K e^{i \beta} z(t-\tau) .
$$

The solution of the Eq.(8) should be given by $z(t)=$ $B e^{(\lambda+i \omega) t}$ where $\lambda$ is a Lyapunov exponent. By substituting the solution into Eq.(8), we have

$$
\lambda=\alpha-K e^{i(\beta-\omega \tau)} e^{-\lambda \tau} .
$$

Here, let $\beta$ compensate the time delay, $\tau$ on the imaginary axis by setting $\beta=\omega \tau[6]$. The equation (9) can be rewritten as

$$
\lambda=\alpha+\frac{1}{\tau} W\left(-\tau K e^{-\alpha \tau}\right)
$$

where $W(x)$ is the Lambert $W$ function, $x=W(x) e^{W(x)}$. As a multivalued function, $W(x)$ has two values in the

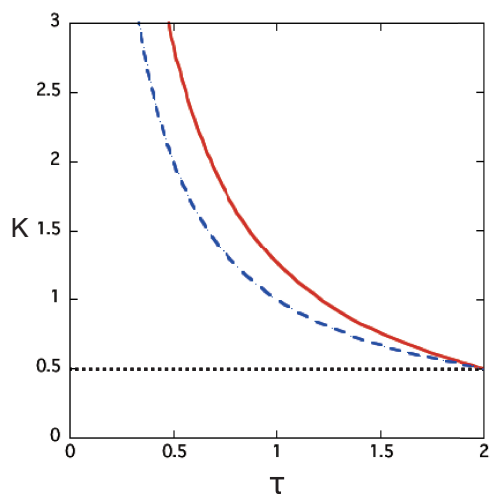

FIG. 1: (Color online) Stability diagram of the anticipating synchronization for the linear system. The solid line represents the case when the delay term, $y(t-\tau)$ is treated exactly. The dashed line represents the case when $y(t-\tau)$ is expanded to the first order. The dotted line $K=\alpha$ represents the stability boundary for both cases $(\alpha=0.5)$. The stability regions are determined by the area surrounded by the solid line and dotted lines for the non-expansion case and by the dashed and doted lines for the case of the first order expansion.

region $-1 / e<x<0$. For $\lambda=0,-\alpha \tau=W\left(-\tau K e^{-\alpha \tau}\right)$ should have solution for $W_{0}(x)$. This condition imposes $-\alpha \tau=W(-1 / e)(=-1)$ which is the extreme value of $W(x)$ function. Thus, the maximum $\tau$ is determined by $\tau>-1 / \alpha$, and $K=\alpha$ is obtained from $x=-1 / e$. The anticipation horizon coincides with the one based on the first order expansion (Fig. 1).

The stability conditions for Lyapunov exponent, $\lambda<$ 0 determines the stability region in $\tau-K$ plane for a given $\alpha$. The solution of Eq. (10) when $\lambda=0$ gives the necessary condition for stability to be $\alpha<K$. For the condition when $\operatorname{Re}(\lambda)=0$, substituting $\lambda=i \theta \quad(\theta \in$ $[0, \infty])$ into Eq. (10) leads to $K=\left(\alpha^{2}+\lambda^{2}\right)^{1 / 2}$ and $\tau=\tan ^{-1}(\theta / \alpha) / \theta$. The stability region of the system is then determined by the region shown in Fig. 1. Note here that the $\tau$ can only give the positive value as a function of $\theta$. Thus, there is no analytical solution for the region $\tau<0$, confirming that Equation (2) cannot refer to the future state of itself.

In contrast to the statement that $x(t+\tau)-y(t)$ is a fixed point of this system for any time delay $\tau$ (the anticipation horizon) [4], the maximum $\tau$ value is given by the intersection of two functions, namely, $K \tau=1$ and $K=\alpha$. This is the same as in the case of stability analysis for the first order expansion, even though the stable area is larger for the driven system on the time delay term.

Thus, from the above analysis, it follows that even explicit account of the feedback delay does not increase the anticipatory horizon compared to the one obtained from the first order expansion. The analytical calculations in this section are restricted to the one dimensional and linear case. We will validate the outcome of the first order 
expansion of the time delay term by numerical calculations in the following sections.

\section{RENORMALIZATION OF TIME}

Let us further consider the outcome of the first order expansion of the time delay term. The expansion of $z(t-$ $\tau)$ up to the first order is equivalent to the expansion of $y(t-\tau)$ in Eq. (2) to the first order, which gives

$$
\frac{d y(t)}{d t^{*}}=f[y(t)]+k[x(t)-y(t)]
$$

where $t^{*}=t /(1-k \tau)$ is a renormalized time in the driven system. From $K>0$ and $\tau>0$ and from Eq. (6), we can obtain $0<1-K \tau<1$, which leads to $t<t^{*}$. For the case of $\tau<0$, we have $1-k \tau>1$, which leads to $t^{*}<t$. This outcome suggests that the time scale of the driven system should be larger or smaller than that of the driver system, depending on the sign of $\tau$. Thus, with respect to the first order expansion, the time delay in the coupling term in Eq. (2) can be equivalently replaced by the renormalized time in the driven system.

\section{NUMERICAL CALCULATIONS FOR MULTIDIMENSIONAL AND NONLINEAR CASES}

\section{A. Rössler chaotic system}

Even though the analytical characterization was obtained only for the one dimensional linear dynamics, the anticipating synchronization based on the renormalized time should be applied to more complex hyperbolic systems. As a test model, we numerically examine the coupled dynamics of a driver and driven system using a Rössler chaotic system. The chaotic oscillator model for the driver system is given,

$$
\begin{aligned}
& \frac{d x_{1}}{d t}=-x_{2}-x_{3} \\
& \frac{d x_{2}}{d t}=x_{1}-a x_{2}, \\
& \frac{d x_{3}}{d t}=b-x_{3}\left(x_{2}-c\right),
\end{aligned}
$$

where $x_{1}, x_{2}$ and $x_{3}$ are state variables. For the driven system based on Eq. (11), we have similarly,

$$
\begin{aligned}
& \frac{d y_{1}}{d t^{*}}=-y_{2}-y_{3}+K\left(x_{1}-y_{1}\right) \\
& \frac{d y_{2}}{d t^{*}}=y_{1}-a y_{2}, \\
& \frac{d y_{3}}{d t^{*}}=b-y_{3}\left(y_{2}-c\right),
\end{aligned}
$$

where $y_{1}, y_{2}$ and $y_{3}$ are state variables of the driven system and $K\left(x_{1}-y_{1}\right)$ represents a coupling between the driver and driven systems. Note that the driven system incorporates the renormalized time scale, $t^{*}=t /(1-k \tau)$ in the time derivative.

Figure 2 shows the numerical results of the Rössler chaotic system when $\tau=0.4$. It is interesting to note that the driven system on the renormalized time scale can show the precedence of phase, even though its amplitude differs more from that of the driver system. Also, shifting the renormalized time for the condition, $t^{*}<t$ when $\tau=-0.4$, the driven system showed the lagging synchronization (Fig. 2). With respect to the first order approximation, we found that the reference to its own future state in the feedback loop actually lead to the delay in response to the driver system. It is intriguing, the the lagging synchronization cannot be achieved by the explicit term in Eq. (2).

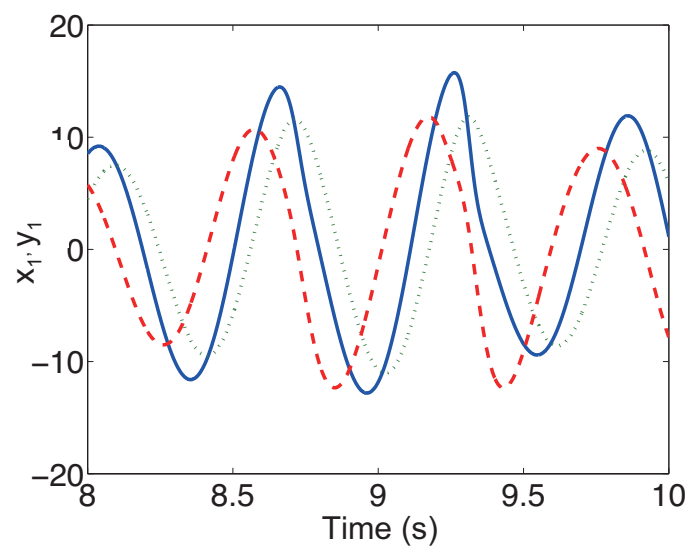

FIG. 2: (Color online) Time series of Rössler chaotic system, $x_{1}(t)$ and $y_{1}(t)$. The solid line represents the driver system with the time step, $\Delta t_{\text {step }}=0.0005$, the dashed line represents the driven system on the renormalized time step, corresponding to $\tau=0.4$, and the dotted line represents the driven system on the renormalized time step, corresponding to $\tau=-0.4$. The values for parameters were $a=0.15, b=0.20, c=10.0, K=1.0$.

Figure 3 shows the stability diagram of the crosscorrelation coefficient with respect to the driver system. The maximum values of the cross-correlation coefficients in the cross-correlation functions were calculated, and the individual values were plotted in $\tau$ - $K$ plane.

For the region $\tau<0.4$ in Fig. 3 (b), the crosscorrelation coefficients are close to 1 , therefore, there is a good agreement between the driver and driven systems in terms of the amplitudes of the curve profiles. However, the cross-correlation coefficients with respect to the driver system decrease gradually as $\tau$ increases more than 0.4 and the area of the good agreement in terms of the amplitude of the curve profile is smaller than the one calculated from the original case of the delay differential equation (Fig. 3 (a)).

Figure 4 shows the stability diagram of the time shift of the driven system, $\triangle t$ with respect to the driver system. 
The time shift is defined by the time lag in the crosscorrelation functions at which the maximum values of the coefficient are to be found. Ideally, the anticipating manifold should be fulfilled as $\triangle t=\tau$ where $\tau$ is a selffeedback delay of the driven system.

However, the driven system based on the renormalized time step shows the larger time shift, $\Delta t>\tau$ for the region of $\tau>0.4$. In this region of $\tau>0.4$, even though the deviation from the driver system becomes larger in terms of amplitude correlation of the functions (Fig. 3), we found the the tendency of the driven system for larger precedence of the driver in the case of the renormalized time step compared to the driven system with the time delay (Fig. 4 (b)).

Note here that the stability diagram obtained by the one dimensional case (Fig. 1) showed a qualitative agreement with the stability diagram of the chaotic system (Fig. 3 and Fig. 4).
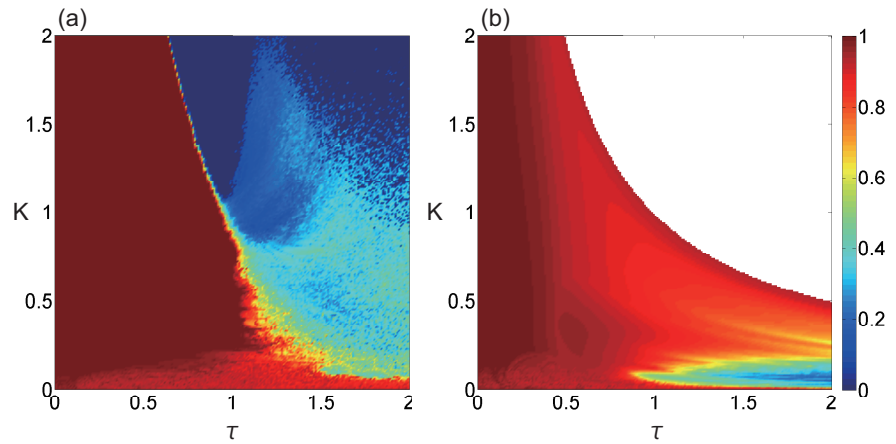

FIG. 3: (Color online) Stability diagram of the crosscorrelation coefficient with respect to the driver system (Rössler system). The maximum values of the coefficient in the cross-correlation functions were plotted in $\tau$ - $K$ plane. The driven systems were based on (a) time delay term (b) renormalized time step. The values of parameters were $a=0.15, b=0.20, c=10.0$.
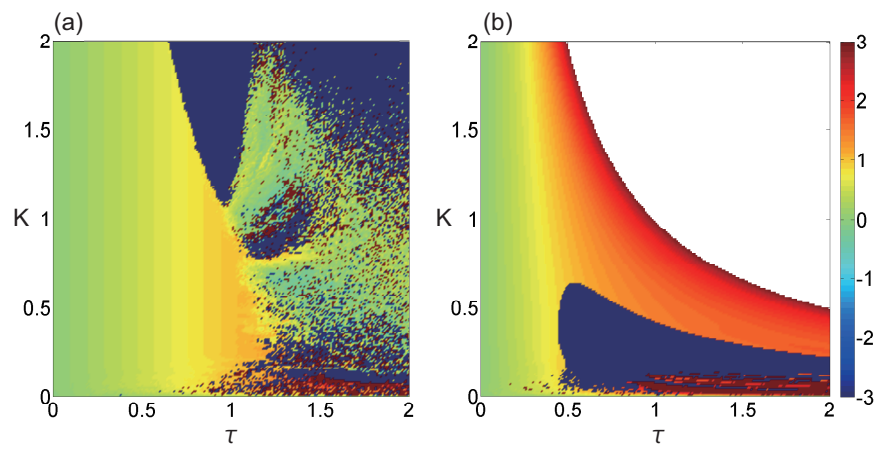

FIG. 4: (Color online) Stability diagram of the time shift with respect to the driver system (Rössler system), which gives the maximum value of the coefficient in the cross-correlation functions. The driven systems were based on (a) Time delay term (b) Renormalized time step. The values of parameters were $a=0.15, b=0.20, c=10.0$.
Figure 5 shows the stability diagram of the lagging synchronization with the positive $\tau$ region. We found that stability region for the negative $\tau$ is consistent with the stability regions derived for the linear case $(K>\alpha)$, and also the smooth transition was found from the lagging to anticipating synchronization as $\tau$ increases from the negative to positive value.
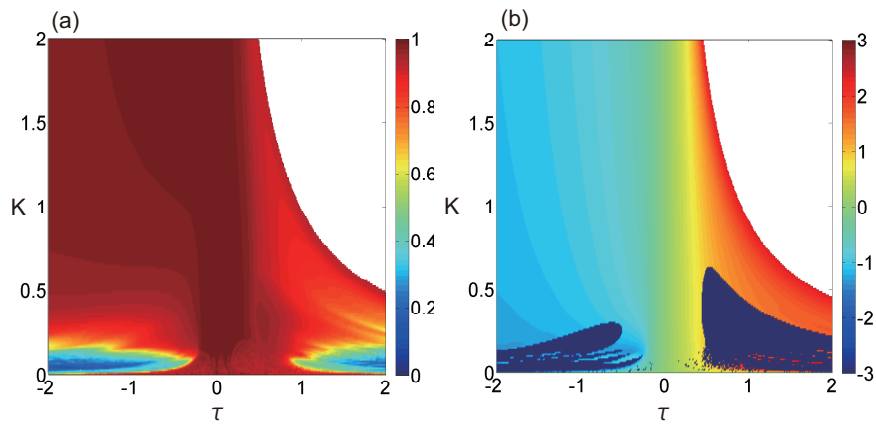

FIG. 5: (Color online) Stability diagram of the lagging synchronization with respect to the driver system (Rössler sys8 tem). The driven systems were based on the renormalized time step. (a) Stability diagram of the maximum cross0.6 correlation coefficient. (b) Stability diagram of the time shift. The values of parameters were $a=0.15, b=0.20, c=10.0$.

\section{B. Excitable systems}

\section{Adler system}

As a next step, we numerically validate that the renormalization of the time step can also work for an excitable system. Adler system is used to study the excitable behavior responding to the same external forcing of the driver and driven system. We consider two identical Adler's systems in unidirectional coupling under the simultaneous external perturbation $I(t)$ on both systems,

$$
\begin{aligned}
\frac{d x}{d t} & =\mu-\cos x+I(t) \\
\frac{d y}{d t^{*}} & =\mu-\cos y+I(t)+K(x-y)
\end{aligned}
$$

where state variable $x$ (driver system) and $y$ (driven system) are angular variables, $\mu$ is the bifurcation parameter. When $|\mu|>0$, the system exhibits the excitable behavior. For the driven system, the time scale is renormalized as $t^{*}=t /(1-K \tau)$.

The excitable systems possess a single stable rest state, corresponding to a fixed point. The small perturbation of the system, for example, the environmental noise pushes the state variable out of the fixed point, and the large excursions of the state variables follow, which are called spikes. Since many biological systems, e.g., neurons, are essentially the excitable systems with a few feedback 


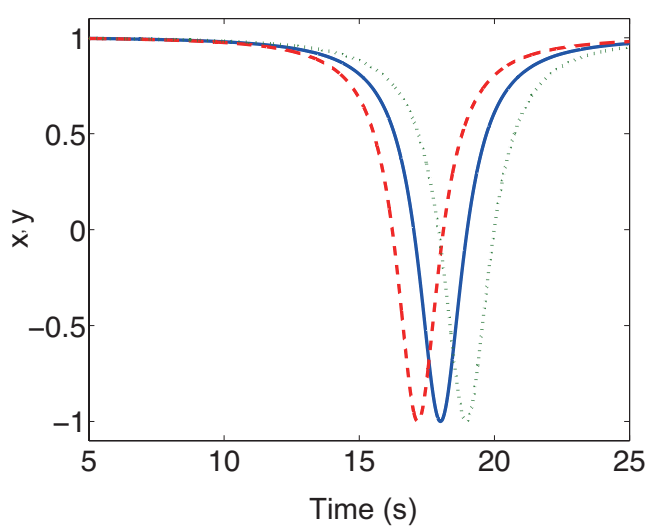

FIG. 6: (Color online) Time series of Adler system, $x(t)$ and $y(t)$. The solid line represents the driver system with the time step, $\triangle t_{\text {step }}=0.0005$, the dashed line represents the driven system on the renormalized time step, corresponding to $\tau=5.0$, and the dotted line represents the driven system on the renormalized time step, corresponding to $\tau=-5.0$. The values of parameters were $\mu=0.95, K=0.01$.

pathways, it is important to consider the excitable system triggered by the random noise to test our hypothesis of the renormalized time. Figure 6 shows that the renormalization of the time step can work in good agreement in terms of amplitude with the driver system for the anticipating synchronization as well as the lagging synchronization.

The driven system based on the renormalized time step showed a wide range of maximum amplitude correlation values with the driven system (Fig.7 (b)), and the degrees of the correlation were almost identical with to those of the driven system based on the time delay feedback (Fig. 7 (a)).

Figure 8 shows the stability diagram of the time shift of the driven system, $\triangle t$ with respect to the driver system. The anticipating manifold should be fulfilled, $\Delta t=\tau$. In the excitable system, the driven system based on the renormalized time step showed the wider stable region fulfilling the anticipating manifold, compared to the chaotic system (Fig. 3 and Fig. 4). Note here that when $\tau>1.5$, the stronger tendency of the time shift was found towards the future state of the driver system, $\Delta t>\tau$.

It is straightforward to understand that the larger time step leads to the higher frequency of the angular state variable. Since the state variable of excitable system, once perturbed to be out of the fixed point, travels the orbit which follows the connection of the saddle and the node. The amplitude of the state variable is well preserved as the state variable travels the orbit. According to the study by Cizak et. Al., the coupling term in the driven system (Eq. (2)) gives rise to the effective $\mu_{e f f}$
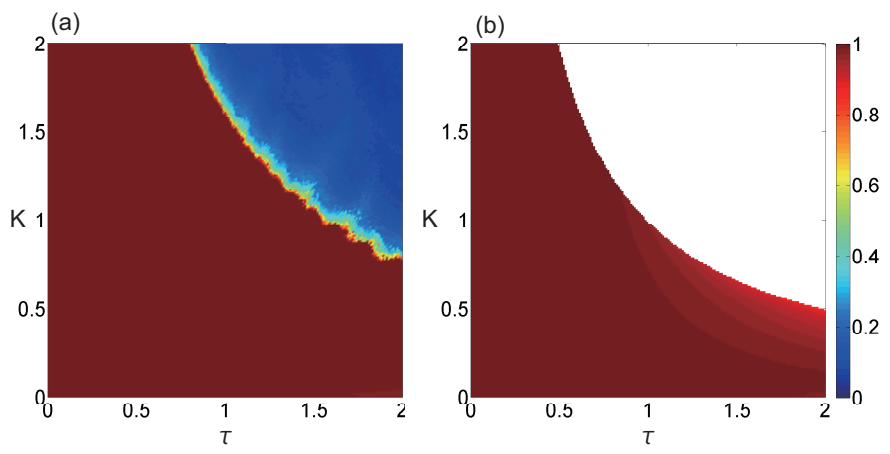

FIG. 7: (Color online) Stability diagram of the crosscorrelation coefficient with respect to the driver system (Adler system). The maximum values of the coefficient in the crosscorrelation functions were calculated in $\tau-K$ plane. The driven systems were based on (a) Time delay term (b) Renormalized time step. The value of parameter was $\mu=0.95$.
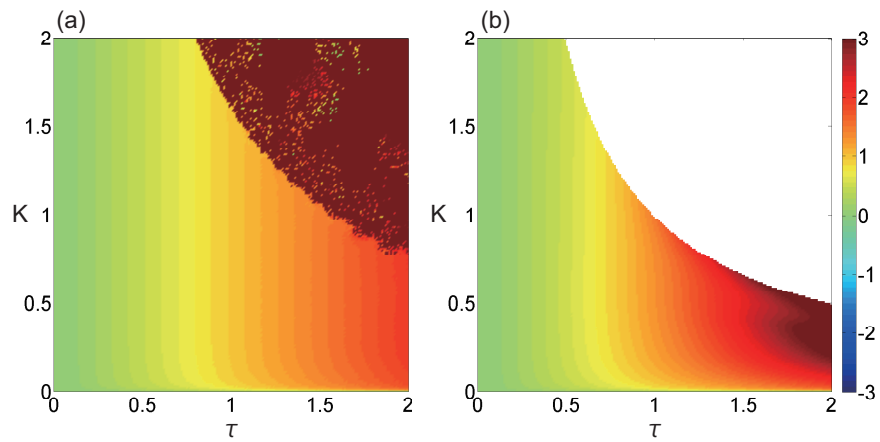

FIG. 8: (Color online) Stability diagram of the time shift with respect to the driver system (Adler system), which gives the maximum value of the coefficient in the cross-correlation functions. The driven systems were based on (a) Time delay term (b) Renormalized time step. The value of parameter was $\mu=0.95$.

$\left(\mu_{\text {eff }}>\mu\right)$ [8], They explained the dynamical mechanism of anticipating synchronization based on the fact that $\mu_{\text {eff }}$ decreases the response time from the onset of perturbation to the time at which the pulse reaches its maximum amplitude. Regarding the first order expansion, substituting $y(t-\tau)$ by $y(t)-\frac{d y}{d t} \tau$ in the coupling term would also lead to the increase of $\mu_{e f f}$ on the onset of perturbation, resulting into the decrease in the response time. Thus, for this Adler system, two explanation, namely, the faster evolution and the effect of $\mu_{e f f}$ on the decrease in the response time would be plausible.

Figure 9 shows the stability diagram of the lagging synchronization with the positive $\tau$ region. We found that the degrees of the correlation were close to one with those of the driver system (Fig. 9 (a)) and that the gradual time shift for the lagging synchronization was also found in the negative $\tau$ region (Fig. 9 (b)). 

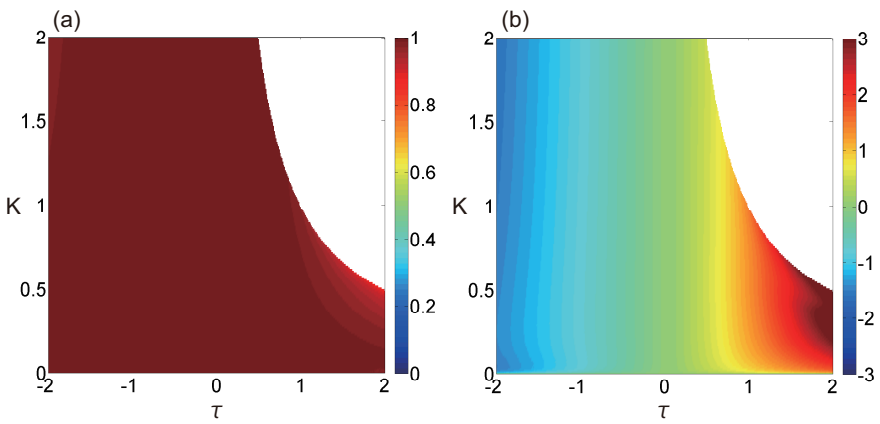

FIG. 9: (Color online) Stability diagram of the lagging synchronization with respect to the driver system (Adler system). The driven systems were based on the renormalized time step. (a) Stability diagram of the maximum cross-correlation coefficient. (b) Stability diagram of the time shift. The values of parameters were $a=0.15, b=0.2, c=10.0$.

\section{FitzHugh-Nagumo system}

The FitzHugh-Nagumo system is another example of a excitable oscillator, a simplified version of the HodgkinHuxley model which models activation and deactivation dynamics of a spiking neuron. This behavior is typical for spike generations in a neuron after stimulation by an external input current. The equations for this dynamical system are given by

$$
\begin{aligned}
\frac{d v_{1}}{d t} & =v_{1}-\frac{1}{3} v_{1}^{3}+I(t) \\
\frac{d w_{1}}{d t} & =\epsilon\left(v_{1}+a-b w_{1}\right)
\end{aligned}
$$

where $v_{1}$ is a membrane voltage, and $w_{1}$ is a linear recovery variable. When the external stimulus exceeds a certain threshold value, the system will be kicked out from the fixed point, and exhibit a characteristic excursion in phase space. On the other hand, the driven system based on the renormalized time, $t^{*}$ is described by

$$
\begin{aligned}
\frac{d v_{2}}{d t^{*}} & =v_{2}-\frac{1}{3} v_{2}^{3}+I(t)+K\left(v_{1}-v_{2}\right) \\
\frac{d w_{2}}{d t^{*}} & =\epsilon\left(v_{2}+a-b w_{2}\right) .
\end{aligned}
$$

Figure 10 shows the numerical results of the FitzHughNagumo system for $\tau= \pm 0.75$. We observe the steady phase shift for the anticipating and the lagging synchronization for the continuous firing. Figure 11 shows the stability diagram of the cross-correlation coefficient with respect to the driver system. When $\tau>1.0$, the driven system starts to deteriorate from dynamics of the driver system. Regarding the stability diagram of the time shift of the driven system (Fig. 12), we found the stronger drive of $\Delta t$ in the region defined by $K<0.2$ and $\tau>0.5$ with respect to the driver system.

Figure 13 shows the stability diagram of the lagging synchronization. We found that the driven system on the

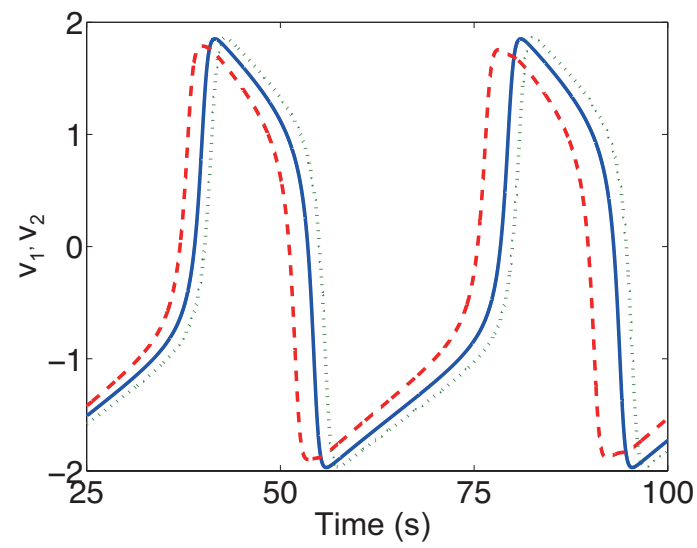

FIG. 10: (Color online) Time series of FitzHugh-Nagumo system, $v_{1}$ and $v_{2}$. The solid line represents the driver system with the time step, $\triangle t_{\text {step }}=0.0005$, the dashed line represents the driven system on the renormalized time step, corresponding to $\tau=0.75$, and the dotted line represents the driven system on the renormalized time step, corresponding to $\tau=-0.75$. The values of parameters were $\epsilon=0.08, a=0.70, b=0.80, K=0.10$.
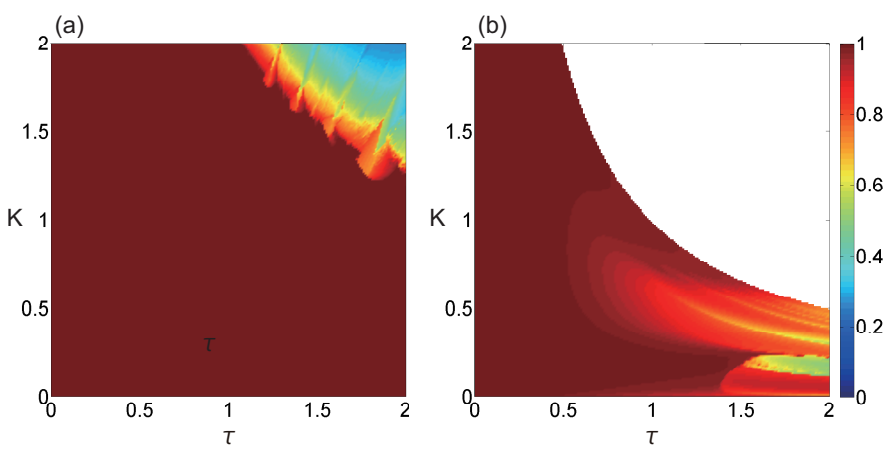

FIG. 11: (Color online) Stability diagram of the crosscorrelation coefficient with respect to the driver system (FitzHugh-Nagumo system). The maximum values of the coefficient in the cross-correlation functions were calculated in $\tau-K$ plane. The driven systems were based on (a) Time delay term (b) Renormalized time step. The values of parameters were $\epsilon=0.08, a=0.70, b=0.80$.

renormalized time scale shows a good agreement with the driven system in terms of amplitude (Fig. 13 (a)) as well as the steady lagging shift (Fig. 13 (b)). 

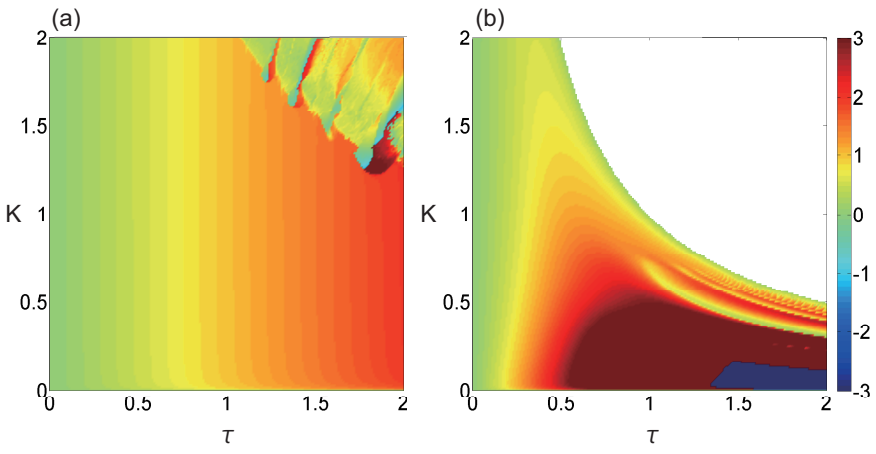

FIG. 12: (Color online) Stability diagram of the time shift with respect to the driver system (FitzHugh-Nagumo system), which gives the maximum value of the coefficient in the crosscorrelation functions. The driven systems were based on (a) Time delay term (b) Renormalized time step. The values of parameters were $\epsilon=0.08, a=0.70, b=0.80$.
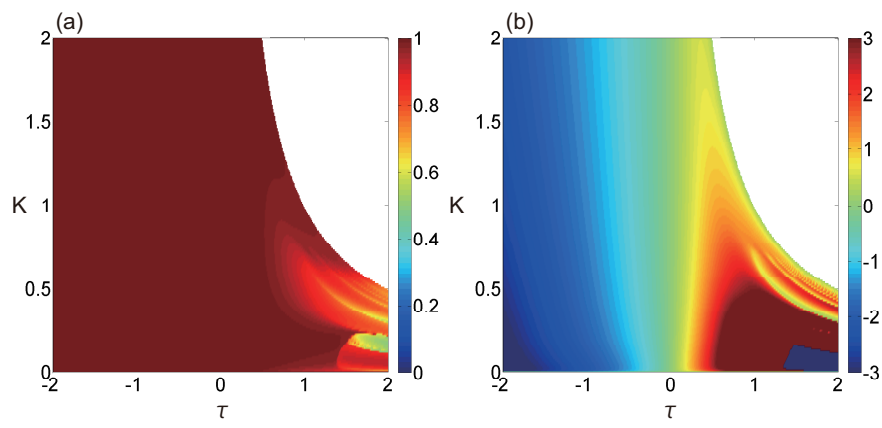

FIG. 13: (Color online) Stability diagram of the lagging synchronization with respect to the driver system (FitzHughNagumo system). The driven systems were based on the renormalized time step. (a) Stability diagram of the maximum cross-correlation coefficient. (b) Stability diagram of the time shift. The values of parameters were $\epsilon=0.08, a=$ $0.70, b=0.80$.

\section{GENERAL DISCUSSION}

Our approach directly links the feedback delay of the driven system with the renormalized time scale of the driven system (Section III), identifying the main component in the anticipating synchronization paradigm, and suggesting an alternative method to generate the anticipating synchronization as well as lagging synchronization.

The following points summarize the method of renormalized time step, extending the paradigm of anticipating synchronization;

1. Larger time scale amounts to the faster evolution of the state variable of the driven system based on the internal dynamics.

2. Smaller time scale amounts to the slower evolution of the state variable of the driven system based on the internal dynamics.
3. Coupling term drives the state variable of the driven systems to be synchronized with the one of the driver system.

4. Interplay between internal dynamics and coupling term gives rise to the steady the anticipating or lagging synchronization of the driven system.

In this study, we proved the ability of anticipating synchronization based on the renormalized time steps in the one dimensional linear case, and systematically validated the outcomes of the renormalized time steps for the case of multidimensional nonlinear dynamics by the numerical calculations. The stability diagram showed that tuning of $\tau$ can induce the anticipating synchronization for $\tau>0$ and the lagging synchronization for $\tau<0$. It is intriguing that the lagging synchronization is only possible in the case of the renormalized time steps.

From the numerically obtained stability diagrams, the renormalized time scale of the driven system was proven to be the alternative method to induce the anticipating synchronization when the time delay term is not available to the driven system. Our results have an important implication that the different time scales of the dynamical systems can be considered as different clocks governing their temporal evolution of the dynamical systems, i.e., depending on the sign of $\tau$, the time evolution of the driven system can be anticipating or lagging with respect to the driver system. When the dynamical systems running based on the different clocks interact unidirectionally, the anticipating synchronization can appear without any time delays. Therefore, our approach will further expand this interesting paradigm towards a wider range of dynamical scenarios.

In behavioural sciences, the feedforward model or the internal predictive model is motivated by the assumption that the feedback delays destabilize the controlled objects. Internal models have been a wide spread concept to explain the human motion overcoming the time-delay in the sensory-motor systems [12]. Kawato concluded that forward internal models can predict sensory consequences from efference copies of issued motor commands and that inverse internal models can calculate necessary feedforward motor commands from desired trajectory information. However, this approach ignores the dynamics of motion, and can be considered as the mapping between the positions in work space and the sequence of motor commands in a static manner.

On contrary to the internal model, Stepp performed tracking experiments in which the participants were given delayed visual feedback of their own movements, and found some characteristics of anticipating synchronization [13]. Even though his finding is at the empirical level, the attempt to emphasize the dynamical coupling with the changing environment should be appreciated to make a link between human behavior and dynamical systems $[14,15]$. Also, in the interpersonal interactions, Hayashi and Kondo found that humans follow the feedback control to tune the cycle time based on the synchro- 
nization errors, and that the gain parameters changes as a function of tapping frequency [16]. From the perspective of renormalization of time, we found that the tuning of the gain parameters can be attributed to the internal time scale to detect the cycle time of tapping motion. Therefore, the internal time scale functioning flexibly to anticipate the changing environment or lag behind it could be a fundamental mechanisms of the living systems for which 'orienting towards the future' is a crucial nature [17].

Using a biologically plausible neuronal model, Matias et. Al. showed that master-slave configuration with the inhibitory neuron can induce anticipating synchronization without the explicit time delay, rather, it was simply triggered by biological plausible delay of the inhibitory neuron to regulate the slave neuron [18]. Recently, iden- tifying the neuronal populations in the cortical regions, the anticipating synchronization was presented as a plausible model for the observed phase shifts in a certain primate cortical circuit under a visual task [19]. This kind of studies can be also explored in terms of the different time scales involved in the individual dynamical systems.

The presence of any feedback delay is regarded as an unfortunate feature of biological and robotic systems. However, as shown in this paper, the positive, productive status of the delayed feedback should be emphasized in terms of the 'internal clock' of the biological systems, as it may have very important implications for our understanding of how neuronal and cognitive systems can predict future outcomes of the changing environment during many crucial tasks from neuronal systems to sensorymotor systems of humans [8, 13, 15, 20, 21].
[1] Y. Kuramoto, Chemical Oscillations, Waves, and Turbulence (Dover Publications, New York, United States, 2003).

[2] A. Pikovsky, M. Rosenblum and J. Kurths, Synchronization: A universal concept in nonlinear sciences (Cambridge University Press, Cambridge, England, 2001).

[3] L. M. Pecora and T. L. Carroll, Phys. Rev. Lett. 64, 821 (1990).

[4] H. U. Voss, Phys. Rev. E, 61, 5115 (2000).

[5] C. Masoller, Phys. Rev. Lett. 86, 2782 (2001).

[6] K. Pyragas and T. Pyragiene, Phys. Rev. E 78, 046217 (2008).

[7] M. Ciszak, O. Calvo, C. Masoller, C. Mirasso, and R. Toral, Phys. Rev. Lett. 90, 204102 (2003).

[8] M. Ciszak, F. Marino, R. Toral, and S. Balle, Phys. Rev. Lett. 93, 114102 (2004).

[9] M. Ciszak, C. R. Mirasso, R. Toral, and O. Calvo, Phys. Rev. E 79, 046203 (2009).

[10] O. Calvo, D. R. Chialvo, V. M. Eguiluz, C. Mirasso, and R. Toral, Chaos, 14, 7 (2004).

[11] N. Corron, J. N. Blakely, and S. D. Pethel, Chaos, 15, $023110(2005)$

[12] M. Kawato, Current Opinion in Neurobiology, 9, 718
(1999).

[13] N. Stepp, Experimental Brain Research, 198, 521 (2009).

[14] N. Stepp and M. T. Turvey, Cogn. Syst. Res. 11-2, 148 (2010).

[15] Y. Hayashi, J. Blake, and S. Nasuto, Anticipation Across Disciplines, M. Nadin (Ed) (Springer, New York, United States, 2015).

[16] Y. Hayashi and T. Kondo, Physical Review E, 88-2, 022715 (2013).

[17] R. Rosen, Anticipatory Systems: Philosophical, Mathematical, and Methodological Foundations (Springer, New York, United States, 2012).

[18] F. S. Matias, P. V. Carelli, C. R. Mirasso, and M. Copelli, Physical Review E 84, 021922 (2011).

[19] F. S. Matias, L. L. Gollo, P. Carelli, S. Bressler, M. Copelli M, C. R. Mirasso, Neuroimage, 99, 411 (2014).

[20] Y. Hayashi and Y. Sawada, Phys. Rev. E, 88, 022704 (2013)

[21] S. Nasuto, S. and Y. Hayashi, Anticipation Across Disciplines, M. Nadin (Ed) (Springer, New York, United States, 2015). 